\title{
Orthogonal Frequency CDMA for Broadband Communications
}

\author{
Shan Tsung Wu and Kwang Cheng Chen \\ Institute of Communications Engineering, and Department of Electrical Engineering \\ National Taiwan University, Taipei, Taiwan 10617 \\ FAX: +886223683824 E-Mail: chenkc@cc.ee.ntu.edu.tw
}

\begin{abstract}
A general scheme called orthogonal frequency CDMA (OFCDMA) to describe CDMA using orthogonal carriers is proposed. Three well-known multicarrier CDMA schemes can be derived as special cases of the proposed OFCDMA if we appropriately define the signature waveforms and separation between adjacent carriers. We proceed a unified analysis of OFCDMA to systematically understand performance of these three schemes. Five types of interference can exist in a OFCDMA system under a multiray multipath fading channel. We compare the three schemes for each type of interference and discuss their pros and cons to help designing effective OFCDMA receivers in the interference-limited environments.
\end{abstract}

\section{Introduction}

CDMA technique has been widely considered for the broadband mobile radio. However, Under the requirement of high transmission data rate, CDMA severely suffers from frequency selective fading that destroys the orthogonality of the signature sequences among each user and results in serious MAI and ISI. Therefore, OFDM technique (or known as multi-carrier) has been proposed combining with CDMA to mitigate this problem while maintaining high bandwidth efficiency by adopting longer symbol duration possibly with guard time and overlapped spectrum. Therefore, the new multiple access schemes based on a combination of DS-CDMA and OFDM techniques have been increasing interest in wireless broadband communications recently [1].

Three different types of such schemes were proposed in literatures. For simplicity, we call these schemes $\mathrm{MC}$ (Multicarrier)-CDMA [2][3][4], Multicarrier DS-CDMA [5] , and MT (multitone)-CDMA.[6]

The MC-CDMA transmitter spreads the original data stream over different subcarriers using a given spreading code in the frequency domain. Figure 1 shows the MC-CDMA transmitter of the $k$ th user for BPSK scheme. Supposing the original data stream is $b_{k}(t)=\sum_{j=-\infty}^{\infty} b_{k}^{\prime \prime} P_{T^{\prime}}\left(t-j^{\prime} T^{\prime}\right)$, where $T^{\prime}$ is the symbol duration of the original data stream and $P_{W}(t)$ is a rectangular pulse of duration $\mathrm{W}$ and unit amplitude. The original data stream is serial-to-parallel (S/P) converted to $J$ groups of data streams $b_{k, j}(t)=\sum_{i=-\infty}^{\infty} b_{k}^{i+t} P_{T}(t-i T)$, where $T$ is the symbol duration of the $\mathrm{S} / \mathrm{P}$ converted data stream. Let $c_{k}^{1} c_{k}^{2} \ldots c_{k}^{M}$ constitute the spreading code for user $k$. Then, $M$ identical branches of data streams of the jth group are spread by the spreading code $c_{k}^{1} c_{k}^{2} \ldots c_{k}^{M}$, and finally each branch modulates a subcarrier $\cos \left(2 \pi\left(\delta \delta_{k, j, m}+f_{1,1}+\left[\frac{1}{T}(j-1)+\frac{J}{T}(m-1)\right]\right) t+\phi_{k, j m}\right)$, i.e. each subcarriers are orthogonal on $T$, where $\phi_{k, j, m}$ is the random phase and, $\delta f_{k, j, m}$ is the frequency deviation. The transmitted signal of user $k$ for MC-CDMA is given by

$s_{k}(t)=\sum_{j=1}^{J} \sum_{m=1}^{M} b_{k, j}(t) c_{k}^{m} \cos \left(2 \pi\left(\delta f_{k, j, m}+f_{1,1}+\left[\frac{1}{T}(j-1)+\frac{J}{T}(m-1)\right]\right) t+\phi_{k, j, m}\right)$

The muiticarrier DS-CDMA transmitter spreads the S/P converted data streams using a given spreading code in the time domain so that the spectrum of each subcarrier be orthogonal with the minimum frequency separation. Figure 2 shows the multicarrier DS-CDMA transmitter of the $k$ th user for BPSK scheme. $M$ identical branches of the $\mathrm{S} / \mathrm{P}$ converted data streams of the $j$ th group is spread by the signature waveform $c_{k}(t)$, and finally each branch modulates a subcarrier $\cos \left(2 \pi\left(\delta \delta_{k, j, m}+f_{t, 1}+\left[\frac{1}{T_{c}}(j-1)+\frac{J}{T_{c}}(m-1)\right]\right) t+\phi_{k, j, m}\right) \quad$ for $\quad m=1,2 \ldots, M$. The transmitted signal of user $k$ for multicarrier DS-CDMA is given by

$s_{t}(t)=\sum_{j=1}^{K} \sum_{m=1}^{M} b_{k, j}(t)_{k}(t) \cos \left(2 \pi\left(\delta \delta_{x, j, m}+f_{1,1}+\left[\frac{1}{T_{c}}(j-1)+\frac{J}{T_{c}}(m-1)\right]\right) t+\phi_{k, j, m}\right)$

The MT-CDMA transmitter spreads the S/P converted data streams using a given spreading code in the time domain so that the spectrum of each subcarrier prior to spreading is orthogonal with the minimum frequency separation. Figure 3 shows the MT-CDMA transmitter of the $k$ th user for BPSK scheme. The S/P converted data streams of the $j$ th group is spread by the signature waveform $c_{k}(t)$, and finally each group modulates a subcarrier $\cos \left(2 \pi\left(\delta f_{k, j}+f_{1}+\frac{1}{T}(j-1)\right) t+\phi_{k, j}\right)$. The transmitted signal of user $k$ for MT-CDMA is given by

$s_{k}(t)=\sum_{j=1}^{5} b_{k, j}(t) c_{k}(t) \cos \left(2 \pi\left(\delta f_{k, j}+f_{i}+\frac{1}{T}(j-1)\right) t+\phi_{k, j}\right)$

Those schemes have been verified to be advantageous over traditional DS-CDMA while the symbol duration in each subcarrier is long enough to yield flat channel fading. However, when the channel is in severe fading, not only the orthogonality of the signature sequences among each user is destroyed but the orthogonality among each carrier also deteriorate as will lead to serious ISI (intersymbol interference), MAI (multiple access interference), and ICI (intercarrier interference). Therefore additional processing such as equalization, joint detection, and multiuser detection may exist for effective receiver design.

\section{OFCDMA scheme}

We propose a general scheme to describe above three multicarrier CDMA by appropriately defining the signature 
waveforms and separation between adjacent carriers. This general scheme is called "orthogonal frequency code division multiple access (OFCDMA)". We further compare and discuss the three different schemes by analyzing MAI, ISI, and ICI of the general scheme, OFCDMA. It is helpful to systematically design the optimum receiver. Furthermore, with this general approach, the pros and cons among the three multicarrier CDMA schemes in different circumstances can also easily be observed for system design in future broadband communications.

Figure 4 shows the OFCDMA transmitter of user $k$ for binary phase shift keying. The original data stream $b_{t}(t)=\sum_{i=-\infty}^{\infty} b_{i}^{i} P_{T}\left(t-j^{\prime} T^{\prime}\right)$ is S/P converted to $J$ groups of data streams $b_{k, j}(t)=\sum_{i=-\infty}^{\infty} b_{k}^{j+j} P_{T}(t-i T) . M$ identical branches of data streams of the $j$ th group are further spread by the signature waveform $c_{k, m}(t)$, and finally each branch modulates a subcarrier $\cos \left(2 \pi f^{\prime}{ }_{k, j, m} t+\phi_{k, j, m}\right) \quad$ for $\quad m=1,2 \ldots M$, where $f_{k, j, m}^{\prime}=f_{j, m}+\delta f_{k, j, m}, \phi_{k, j, m}$ is the random phase, and $\delta f_{k, j, m}$ is the frequency deviation. The transmitted signal of user $k$ is given by $s_{k}(t)=\sum_{j=1}^{J} \sum_{m=1}^{M} b_{k, j}(t) c_{k, m}(t) \cos \left(2 \pi f^{\prime}{ }_{k, j, m} t+\phi_{k, j, m}\right)$

$$
=\sum_{j=-\infty}^{\infty} \sum_{m=1}^{M} \sum_{i=-\infty}^{\infty} b_{k}^{j+j} P_{\tau}(t-i T) c_{k, m}(t) \cos \left(2 \pi f^{\prime}{ }_{k, j, m} t+\phi_{k, j, m}\right)
$$

Now we are ready to show that the three multicarrier CDMA schemes are just special cases of OFCDMA in Figure 4.

In (4), If the signature waveforms are designed such that $c_{k, m}(t)=c_{k}^{m}$, where $c_{k}^{1} c_{k}^{2} \ldots c_{k}^{M}$ constitute a PN (pseudo-noise) sequence for user $k$ and $T=M J T^{\prime}$, and if subcarriers are orthogonal on $T$, i.e. frequency separation satisfies $f_{a, b}-f_{c, d}=\frac{1}{r}(a-c)+\frac{J}{T}(b-d)$, then (4) is reduced to (1) which is obviously the transmitted signal of user $k$ for MC-CDMA.

In (4), if the signature waveform is such that each subcarrier of user $k$ has the same signature waveform i.e. $c_{k, m}(t)=c_{k}(t)=\sum_{n^{\prime}=-\infty}^{\infty} c_{k}^{n} P_{T_{c}}\left(t-n^{\prime} T_{c}\right)$, where $n=1+\left(n^{\prime}-1\right) \bmod N$, $T_{c}=\frac{T}{N}, c_{k}^{1} c_{k}^{2} \ldots . c_{k}^{N}$ constitute a PN sequence for user $k$ and $T=J T^{\prime}$, and if the subcarriers are orthogonal on $T_{c}$, i.e. frequency separation satisfies $f_{a, b}-f_{c, u}=\frac{1}{T_{c}}(a-c)+\frac{J}{T_{c}}(b-d),(4)$ is reduced to (2) which is obviously the transmitted signal of user $k$ for multicarrier DS-CDMA.

In (1), if there is only one branch in each group $j$, i.e. $M=1$ $c_{k, 1}(t)=c_{k}(t)=\sum_{n=-\infty}^{\infty} c_{k}^{n} P_{T_{c}}\left(t-n^{\prime} T_{c}\right), n=1+\left(n^{\prime}-1\right) \bmod N_{M T}, T_{c}=\frac{T}{N_{M T}}$, and $T=J T^{\prime}$, where $c_{k}^{1} c_{k}^{2} \ldots c_{k}^{N_{M r}}$ constitute a PN sequence for user $k$, $T=J T^{\prime}$. However, the subcarriers are orthogonal on $T$ i.e. frequency separation satisfies $f_{a}-f_{b} \equiv f_{a, 1}-f_{b, 1}=\frac{1}{T}(a-b),(4)$ is reduced to (3) which is obviously the transmitted signal of user $k$ for MT-CDMA.

\section{Interference analysis}

We proceed to study OFCDMA in multiray multipath fading channels. Suppose the complex low-pass impulse response of the channel for carrier $(j, m)$ of the $k$ th user is: $h_{k, j, m}(t)=\sum_{t=1}^{L} g_{k, j, m, t} \delta\left(t-t_{k, j}\right)$

where $L$ is the number of resolvable paths, and $g_{k, j, m, l}=\beta_{k, j, m, t} e^{\gamma_{k, j-s}}$ is a complex Gaussian r.v. (random variable) with zero mean and variance $\sigma_{l}^{2}$, i.i.d. (identical and independent distribution) for all $k, j, m, t_{k, l}=(l-1) T_{c}+\Delta_{k, l}$ is the delay of the $l$ th path of the $k$ th user. We assume $\Delta_{k, l}$ are identical and independent r.v.s uniformly distributed on $\left[0, T_{c}\right)$ for all $k$ and $l$ denoted by $U\left[0, T_{c}\right)$ for simplicity. With the assumption of perfect power control at the receiver in asynchronous case, the received signal is as (6):

$v(t)=\sqrt{2 p} \sum_{k=1}^{K} \sum_{i=1}^{L} \sum_{j=1}^{K} \sum_{n=1}^{M} \beta_{k, j, m, j} b_{k, j}\left(t-t_{k j}-\tau_{k}\right) c_{k, m}\left(t-t_{k, j}-\tau_{k}\right) \cos \left(2 \pi f^{\prime}{ }_{k, j, m} t+\varphi_{k, j, m}\right)+\eta(t)$

where $\eta(t)$ is AWGN, $\varphi_{k, j, m, j}=\left(\phi_{k, j m}+\gamma_{k, j, j}-2 \pi f_{k, j, m}^{\prime}\left(t_{k j}+\tau_{k}\right)\right) \bmod 2 \pi$, and $\tau_{k}$ is the propagation delay for user $k$. The sets $\left\{\varphi_{k, j, m,}\right\}$ and $\left\{\tau_{k}\right\}$ are i.i.d. $U[0,2 \pi)$ and $U[0, T)$ respectively. Suppose the receiver consists of a $\lambda$-fingered RAKE receiver for each carrier. Without loss of generality, we assume the receiver of user 1 is the intended one and let $\tau_{1}=0$. The decision variable $Z_{r, s, t}$ at the output of the MF (matched filter) for the zeroth bit on branch $s$ of group $r$ of user 1 that locks to the $n$th ray is:

$Z_{r, s, n}=\int_{t, .,}^{1, x+r} v(t) c_{1, s}\left(t-t_{1, n}\right) \cos \left(2 \pi f_{r, s} t+\varphi_{1, r, s, n}\right) d t$

We assume the decision r.v.s of the MF are maximal ratio combined for $s=1,2 \ldots, M$. The combined decision variable $Z^{r}$ is the output for the zeroth bit of $b_{1, r}(t)$.

$Z^{r}=\sum_{s=1}^{M} \sum_{n=1}^{A} \beta_{1, r, s, n} Z_{r, s, n}=\eta+D^{r}+I^{r 1}+I^{r 2}+I^{r^{3}}+I^{44}+I^{r s}$

where $\eta$ is due to AWGN and is a zero mean Gaussian r.v, with variance $\frac{N_{0} T}{4}$. Without loss of generality, we assume $\tau_{1}=0$, $\varphi_{1, r, s, n}=0$, and $\Delta_{1, n}=0, D^{r}$ in (7) is the desired signal output corresponding to $l=1, j=r, m=s$ at the summation index in (6).

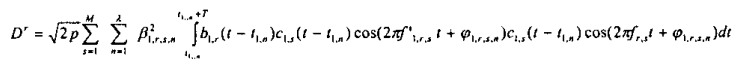

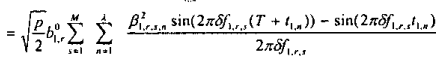

In addition, there are five types of uncorrelated interference in (7). Please note that those types of interference are zero mean due to random bits. We use well-know Gaussian approximation for our analysis of bit error rate (BER) of OFCDMA which provides satisfactory results when the numbers of users are large and $P N$ sequence is long enough. In Gaussian analysis of BER for CDMA, we only need to know signal to interference ratio. Therefore we are going to find the variances for all types of interference. Since all types of interference have zero mean, the variances of them are just the mean square.

A ) $I^{r !}$, ISI due to other rays $l \neq n$ on the same carrier $(j, m)=(r, s)$ from the same user $k=1$ in (6)

This type of interference is a result of time shift of other symbols from other rays. $I^{r 1}$ is given by

$I^{\prime \prime}=\sqrt{\frac{p}{2}} \sum_{s=1}^{M} \sum_{n=1}^{L} \sum_{\substack{i=1 \\ s=1}}^{L} I_{s, n, t}^{r 1}$ 
where

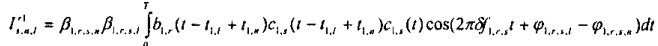

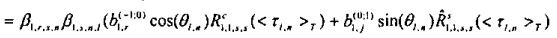

here we define $\langle x\rangle_{T} \equiv x \bmod T, \quad \tau_{l, n}=t_{1, l}-t_{1, n}$, $\theta_{l, n}=\varphi_{1, r, s, l}-\varphi_{1, r, s, n}$ for convenience. The continuous time partial weighted crosscorrelation functions in (9) are defined by

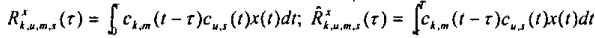

$x(t)= \begin{cases}\cos (w t) & \text { when } x=c \\ \sin (w t) & \text { when } x=s \\ 1 & \text { when } x=1\end{cases}$

$x(t)=\left\{\begin{array}{lr}\sin (w t) & \text { when } x=s \\ 1 & \text { when } x=1\end{array}\right.$

$w=2 \pi\left(\delta f_{h, j, m}+f_{j, m}-f_{r, s}\right)$ in general. Because $\sin (\varphi)$ and $\cos (\varphi)$ are orthogonal for $\varphi \sim U[0,2 \pi)$, the two terms in (9) are orthogonal. In (8), all terms inside the summation are orthogonal except for some terms that incorporate the same phase angle with opposite polarity (for example, at $l=1$ and $n=2$, $\sin \left(\theta_{l, n}\right)=-\sin \left(\theta_{n, l}\right)$ at $l=2$ and $n=1$ ). We combine correlated terms in (9) such that all terms constituting $I^{r i}$ are orthogonal for different summation indexes as much as possible. Then the variance of sum equals the sum of variances if all terms with different summation indexes are orthogonal to each other. After taking the second moment of (9) over the random angles, Rayleigh r.v.s, random bits and $\tau_{i, n}$, we get

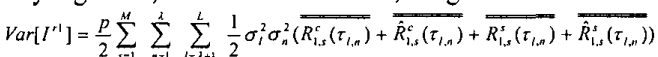

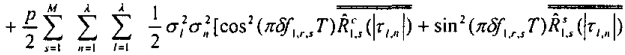

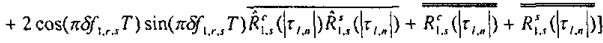

where $\overline{(.)} \equiv E[$.$] and \overline{\overline{(.)}} \equiv E\left[(.)^{2}\right]$. Note that the first term in the RHS (right hand side) of (12) vanishes when $\lambda=1$ (nonRAKE )

B ) $I^{r 2}$, the ICI due to the same ray $l=n$ on other carriers $(j, m) \neq(r, s)$ from the same user $k=1$ in (6)

This type of interference is a result of frequency deviation $\delta f_{1, j, m}$ which destroys the orthogonality among carriers. $I^{r 2}$ is given by

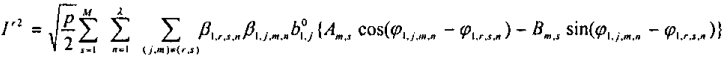
where

$A_{m, s}=\int_{0}^{r} \cos \left(2 \pi\left(\delta f_{1, j, m}+f_{j, m}-f_{r, s}\right) t\right) d t ; \quad B_{m, s}=\int_{0}^{T} \sin \left(2 \pi\left(\delta f_{1, j, m}+f_{j, m}-f_{r, s}\right) t\right) d t$

With the same method to calculate the variance of sum described in $A$ ), we get

$\operatorname{Var}\left[I_{r}^{2}\right]=\left.\frac{p}{2} \sum_{s=1}^{M} \sum_{n=1}^{\lambda} \sum_{\substack{m=1 \\ t s}}^{M} \frac{\sigma_{n}^{4}}{2}\left\{\left(A_{m, s}+A_{s, m}\right)^{2}+\left(B_{m, s}+B_{s, m}\right)^{2}\right\}\right|_{j=r}$

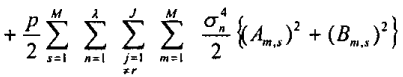

Not that when there are no frequency deviations, this type of interference disappears. It is actually an important issue in future work.

C ) $I^{r 3}$, the ICI due to other rays $l \neq n$ on other carriers $(j, m) \neq(r, s)$ from the same user $k=1$ in $(6)$

This type of interference is caused by frequency deviation $\delta f_{1, j, m}$ and ISI on each carrier (due to multiray) that destroys the orthogonality among carriers. $I^{r 3}$ is given by

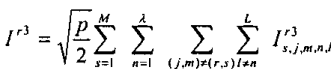

where

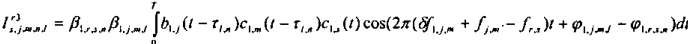

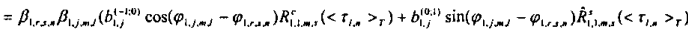

With the same method to calculate the variance of sum described in $A)$, we get

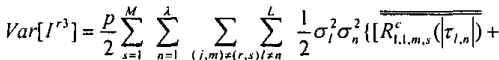

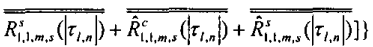

$+\frac{p}{2} \sum_{i=1}^{M-1} \sum_{n=1}^{R-1} \sum_{j=r} \sum_{m=s+1}^{M} \sum_{i=n+1}^{R} \frac{1}{2} \sigma_{i}^{2} \sigma_{n}^{2}\left\{\left(E\left[\cos \left(2 \pi \delta f_{1, r, m} \tau_{l, n}\right)\right]\right.\right.$

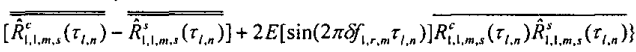

D ) $I^{r 4}$, the conventional MAI due to all rays from the same carrier $(j, m)=(r, s)$ from other users $k \neq 1$ in (6)

This type of interference is mainly due to cross correlation of signature waveforms (ISI on each signature waveform) among different users

$I^{r 4}=\sqrt{\frac{p}{2}} \sum_{k=2}^{K} \sum_{s=1}^{M} \sum_{n=1}^{\lambda} \sum_{i=1}^{L} I_{k s, a, t}^{r 4}$

where

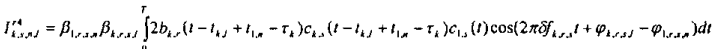

Since $\left(t_{k, l}-t_{1, n}+\tau_{k}\right) \bmod T$ and $\left(\tau_{k}+t_{k, l}\right) \bmod T$ are i.i.d. for all $k$ respectively. There is no loss of generality in setting $t_{1, n}=0$, i.e. the $n$th path of user one is the time reference for other users. All terms constituting $I^{r 4}$ for different summation indexes are orthogonal since they incorporate independent phases. After taking the second moment of $I^{r 4}$, we have

$E\left[I^{44}\right]=\frac{p}{2} \sum_{k=2}^{K} \sum_{s=1}^{M} \sum_{n=1}^{\lambda} \sum_{i=1}^{L} \frac{\sigma_{n}^{2} \sigma_{l}^{2}}{2}\left\{\overline{\left\{\overline{R_{k, 1, s, s}^{c}\left(t_{k, t}+\tau_{k}\right)}\right.}\right.$

$+\overline{\overline{R_{k, k s, s}^{s}\left(t_{k, j}+\tau_{k}\right)}}+\overline{\overline{\hat{R}_{k, 1, s, s}^{c}\left(t_{k, j}+\tau_{k}\right)}}+\overline{\overline{\left.\hat{R}_{k, 1, s, s}^{s}\left(t_{k, 1}+\tau_{k}\right)\right\}}}$

E ) $I^{r 5}$, the multicarrier MAI due to all rays from other carriers $(j, m) \neq(r, s)$ from other users $k \neq 1$ in (6)

This type of interference is the MAI from other carriers and does not exist for conventional CDMA. It is mainly caused by destruction of orthogonality on signature waveforms (ISI on each signature waveform due to multiray) among different users. And destruction of orthogonality among carriers (ICI on each carrier due to multiray). With the same method as that used in calculating the variance of $I^{r 4}$, we have

$$
\begin{aligned}
& \operatorname{Var}\left[I^{r s}\right]=\frac{p}{2} \sum_{k=2}^{K} \sum_{s=1}^{M} \sum_{n=1}^{\lambda} \sum_{(j, m) \neq(r, s) l=1} \sum_{t=1}^{L} \frac{\sigma_{n}^{2} \sigma_{l}^{2}}{2} \overline{\left(\overline{R_{k, 1, m, s}^{c}\left(t_{k, l}+\tau_{k}\right)}\right.} \\
& +\overline{\overline{R_{k, 1, m, s}^{s}\left(t_{k, l}+\tau_{k}\right)}}+\overline{\overline{\hat{R}_{k, 1, m, s}^{c}\left(t_{k, l}+\tau_{k}\right)}}+\overline{\overline{\hat{R}_{k, 1, m, s}^{c}\left(t_{k, l}+\tau_{k}\right)}}
\end{aligned}
$$

\section{Discussion and comparison of the multicarrier CDMA}

The importance of each type of interference in OFCDMA can be determined by the numerical value of its variance. This information is 
useful for designing effective receivers. For example, if some interference dominates, an intuitive way to design the receiver is try to eliminate the dominated one. We are further interested in the selection of multicarrier CDMA schemes to for certain application scenarios. We are going to systematically discuss the performance of the three schemes by comparing the variances of each type of interference among MC-CDMA, multicarrier DS-CDMA, and MT-CDMA respectively. It is obvious from (12) (14) (15) (16) that the variances for all types of interference, except the ICI due to frequency deviation $I^{r^{2}}$ primarily consist of $\overline{R_{x, \mu, n, s}^{x}[l]}$ and ${\hat{R_{k, u, m s}}}_{x}[l]$, the mean square of the continuous time partial weighted crosscorrelation functions defined in (10). Assuming $t=l T_{c}+\Delta, 0 \leq l \leq N-1$, where $\Delta-U\left[0, T_{c}\right]$, and from $(10)$ we get

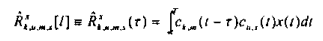

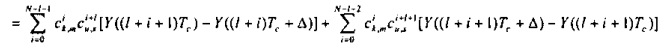

where $Y(t)=\int x(t) d t, x(t)=\sin (w t)$ or $x(t)=\cos (w t)$.

With the use of triangular identities (17) becomes

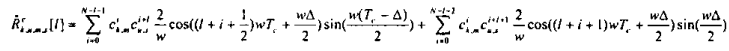

The random term $\frac{m \Delta}{2}$ inside $\cos ($.) can be considered as a part of the random phase of carriers, and can be neglected. With the help of triangular identities we can obtain the mean square of $\hat{R}_{k, u, m, s}^{x}[l]$ as

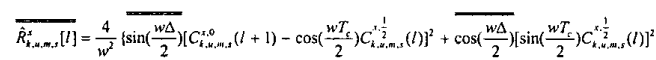

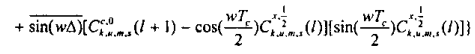

where the aperiodic weighted crosscorrelation function $C_{x, u, m, s}^{x, a}(l)$ for the sequence $c_{k, m}^{i}$ and $c_{u, s}^{\prime}$ is defined by

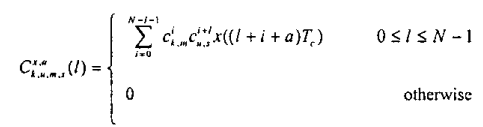

With similar method, it can also be shown that.

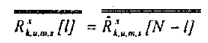

In the following, we will not consider the effect of frequency deviation on all carriers, i.e. $\delta f_{k, j, m}=0$. The randomness for each ray in multipath fading channel is modeled by $\Delta \sim U\left[0, T_{c}\right)$. We will assume $\Delta=0$, i.e. no randomness for each ray, for ease of comparison by directly

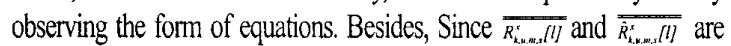
symmetric about $\frac{N}{2}$, we can only consider $\overline{\overline{\hat{R}_{x, \text { m s }}^{*}(l)}}$ when making

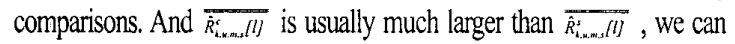
only consider the importance of $\overline{\hat{R}_{\text {inas }}^{c}(l l}$ among the schemes. With the two assumptions and setting $x=c$ in (18), then (18) becomes (19)

$\overline{\dot{R}_{i, \ldots, \ldots}^{c}[l]}=T_{r}^{2} \operatorname{sinc}\left(\frac{w T_{c}}{2}\right)\left[C_{t, \ldots, \ldots, s}^{r \cdot \frac{1}{2}}(l)\right]^{2}$

\section{A) $I^{\prime \prime}$, the ISI due to other rays in multipath fading channels}

Let's consider $I^{r}$ first. The parameter $w$ in $(10)$ and (20) is zero in this case. Let $\left.\right|_{M C},\left.\right|_{M D},\left.\right|_{M T}$ denote the condition on MC-CDMA, multicarrier DS-CDMA, and MT-CDMA respectively. For MC-CDMA

$$
\left.\overline{\overline{\hat{R}_{1,1, s, s}^{c}[l]}}\right|_{M C}=T_{c}^{2}\left[\sum_{i=0}^{N-1-1} c_{1}^{s} c_{1}^{s}\right]^{2}=T_{c}^{2}[N-l]^{2}
$$

Note that (20) is independent of its PN-sequence. $\left\{c_{1, m}^{i} \equiv c_{1}^{m}\right\}_{m=1,2 \ldots, m}$.
For both multicarrier DS-CDMA and MT-CDMA

$\left.\overline{\overline{\hat{R}_{1,1, s, s}^{c}[l]}}\right|_{M D}=\left.\overline{\overline{\hat{R}_{1,1, s, s}^{c}[l]}}\right|_{M D}=T_{c}^{2}\left[\sum_{i=0}^{N-l-1} c_{1}^{i} c_{1}^{i+l}\right]^{2}$

Note that (21) is related to the aperiodic autocorrelation function of the PN-sequence $\left\{c_{1, m}^{i}=c_{1}^{i}\right\}_{i=1,2 \ldots, N}$. It is obvious from (20) and (21) that MC-CDMA has larger $I^{r l}$ than multicarrier DS-CDMA and MT-CDMA

B) $I^{2}$, the ISI due to frequency deviation and uncertainty

$\operatorname{Var}\left[I^{r^{2}}\right]=0$ for all schemes in case of no frequency deviation.

C) $I^{r 3}$, the ICI

$\left.\overline{\overline{\hat{R}_{1,1, m, s}^{c}[l]}}\right|_{M C}=T_{c}^{2} \operatorname{sinc}\left(\frac{w T_{c}}{2}\right)^{2}\left[c_{1}^{m} c_{1}^{s} \sum_{i=0}^{N-i-1} \cos \left((l+i+a) w T_{c}\right)\right]^{2}$

which is related to the weighted partial energy of the sequence $\left.\left\{c_{1}\right\}\right\}_{i=1,2, \ldots, \mu}$. For multicarrier DS-CDMA

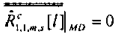

since $w T_{c}=2 \pi[(j-r)+J(m-s)]$, we have $\sin \left(\frac{w T_{c}}{2}\right)=0$. This is not surprising because we neglect the effect of the $\Delta$.And for MT-CDMA

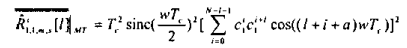

which is related the aperiodic weighted autocorrelation function of $\left\{c_{1}\right\}_{i=1,2, \ldots, M}$. Note that $w T_{c}=\frac{2 \pi}{N}[(j-r)+J(m-s)]$ for both MCCDMA and MT-CDMA. Because $\left(c_{1}^{m} c_{1}^{s}\right)^{2}=1$ it is obvious that $I^{r 3}$ is larger in MC-CDMA than that in MT-CDMA.

D) $I^{r 4}$, the conventional MAI

Then, let's consider $I^{r 4}$. The parameter $w$ in (10) and (20) is zero in this case. For MC-CDMA

$\left.\overline{\overline{\hat{R}_{k, 1, s, s}^{c}[l]}}\right|_{M C}=T_{c}^{2}\left[\sum_{i=0}^{N-l-1} c_{K}^{S} c_{1}^{S}\right]^{2}=T_{c}^{2}[N-l]^{2}$

For both multicarrier DS-CDMA and MT-CDMA

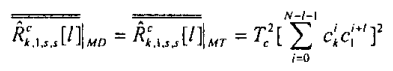

which is related to the aperiodic crosscorrelation function of the PN-

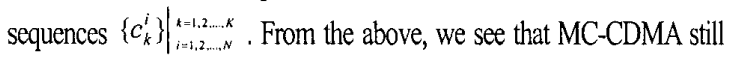
has the largest $I^{r 4}$.

E) $I^{r 5}$, the multicarrier MAI

Finally let's consider $r^{r s}$. For MC-CDMA

$\left.\overline{{\hat{R_{k, l, m, s}^{c}}}_{[}[l]}\right|_{M C}=T_{c}^{2} \operatorname{sinc}\left(\frac{w T_{c}}{2}\right)^{2}\left[\sum_{i=0}^{N-l-1} \cos \left((l+i+a) w T_{c}\right)\right]^{2}$

which is independent of the PN-sequences. for multicarrier DS-CDMA

$\left.\overline{\hat{R}_{k, 1, m, s}^{c}[l]}\right|_{M D}=0$

since $w T_{c}=2 \pi[(j-r)+J(m-s)]$, then ${ }^{\sin \left(\frac{w T_{c}}{2}\right)=0}$.

For MT-CDMA

$\left.\overline{\overline{\hat{R}_{k, 1, m, s}^{c}[l]}}\right|_{M T}=T_{c}^{2} \operatorname{sinc}\left(\frac{w T_{c}}{2}\right)^{2}\left[\sum_{i=0}^{N-l-1} \cos \left((l+i+a) w T_{c}\right)\right]^{2}$

which is related to the aperiodic weighted crosscorrelation function of 


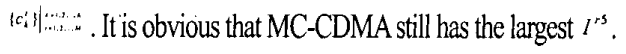

It seems so surprising that MC-CDMA has the worst performance among the three. However this is not completely true! Since in our analysis we assume that each carrier has independent random phases, it only suggests that MC-CDMA is less favorable for incoherent modulation. MC-CDMA cannot take advantage of the properties of PNsequences to reduce two types of interference, $I^{r 1}$ and $I^{r 4}$. In fact, it can be shown that MC-CDMA has smallest $I^{r 1}$ and $I^{r 4}$ among the three if in coherent modulation. Next let us compare between multicarrier DSCDMA and MT-CDMA. Both schemes differ primary in the separation among carriers. They have equal quantity of $I^{r 1}$ and $I^{r 4}$, but MT-CDMA has larger $I^{r 3}$ and $I^{r 3}$ than multicarrier DS-CDMA, this is the disadvantage of smaller carner separations. However, MT-CDMA indeed has the advantage of better bandwidth efficiency. There exits a trade-off between them.

\section{Conclusion}

A general scheme OFCDMA to describe CDMA using orthogonal carriers was proposed. Three well-known schemes can be considered as special cases of OFCDMA. We made an analysis of the performance of OFCDMA in a multiray Rayleigh fading channel, and then discussed the pros and cons of OFCDMA with different specification of parameters for MC-CDMA, multicarrier DS-CDMA, and MT-CDMA. It was shown that MC-CDMA is less favorable for incoherent modulation. Multicarrier DS-CDMA and MT-CDMA have equal quantity of $I^{r \prime}$ (ISI) and $I^{r 4}$ (MAI), but the latter has larger $I^{r 3}$ (ICI) and $I^{r s}$ (multicarrier MAI) than the former due to smaller separation among carriers. However, MTCDMA has the advantage of better bandwidth efficiency. There is a trade-off in BER and bandwidth efficiency in OFCDMA.

\section{References:}

[1] S. Hara and R. Prasad, "Overview of multicarrier CDMA," IEEE Commun. Mag., Dec. 1997, pp. 126-33.

[2] N. Yee, J-P. Linnartz and G Fettweis, "Multicarrier CDMA in Indoor Wireless Radio Networks," Proc. Of IEEE PIMRC '93, Yokohama, Japan, Sept. 1993, pp. 109-13

[3] K. Fazel and L. Papke, "On the Performance of convolutionally-coded CDMA/OFDM for Mobile Communication System," Proc. Of IEEE PIMRC'93, Yokohama, Japan, Sept. 1993, pp468-72.

[4] A. Chouly, A. Brajal and S. Jourdan, "Othogonal Multicarrier Techniques Applied to Direct Sequence Spread Spectrum CDMA Systems," Proc. Of IEEE GLOBECOM '93, Houston, USA, Nov. 1993, pp. 1723-28.

[5] V. M. DaSilva and E. S. Sousa, "Performance of Orthogonal CDMA Codes for Quasi-Synchronous Communication Systems," Proc. of EEE ICUPC' '93, Ottawa, Canada, Oct. 1993, pp.995-99.

[6] Vandendorpe, "Multitone Direct Sequence CDMA System in an indoor Wireless Environment," Proc. of IEEE First Symposium of Communications and Vehicular Technology in the Benelux, Delft, The Netherlands, Oct. 1993, pp. $4.1-1-4.1 .8$.

[7] S. Hara, T-H. Lee and R. Prasad, "BER Comparison of DS-CDMA and MCCDMA for Frequency Selective Fading Channels, " Proc. of $7^{\text {th }}$ Tyrrhenian Intemational Workshop on Digital Communications, Viareggio, Italy, Sept. 1995, pp3-14.

[8] E. A. Sourour, and M. Nakagawa, "Performance of Orthogonal Multicarrier CDMA in a Multipath Fading Channel," IEEE Trans. Commun., Mar. 1996, pp.356-67.

[9] L. Vandendorpe "Multitone Spread Spectrum Multiple Access Communications System in a Multipath Rician Fading Channel" IEEE Trans.
Commun., May. 1995, pp.327-37.

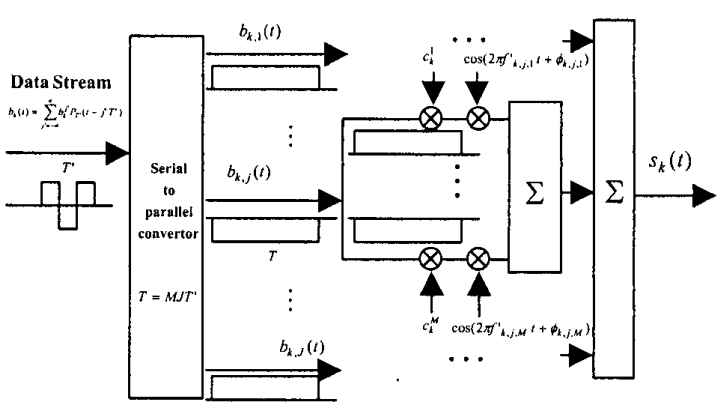

Figure 1 The MC-CDMA scheme

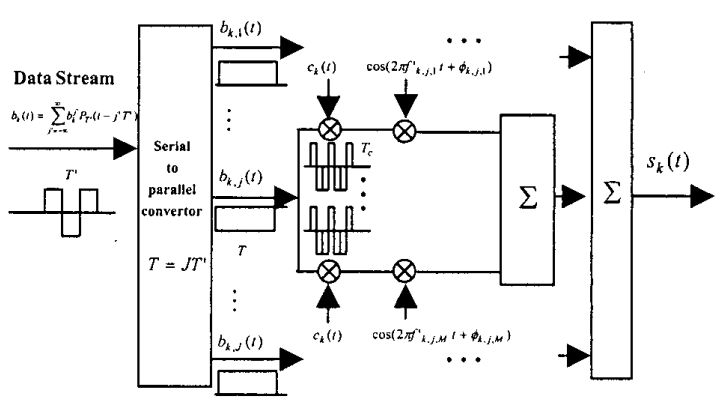

Figure 2 multicarrier DS-CDMA scheme

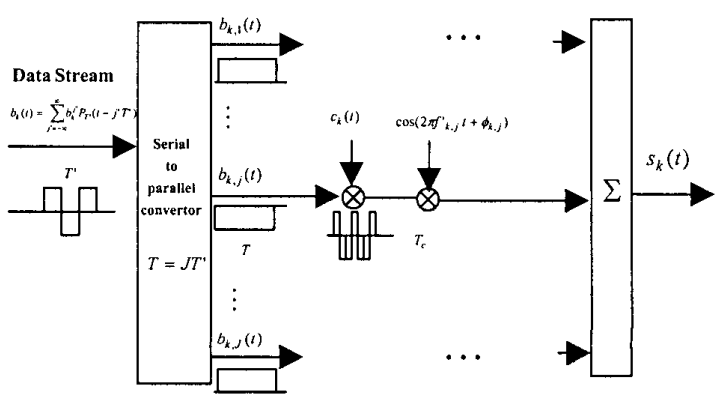

Figure 3MT-CDMA scheme

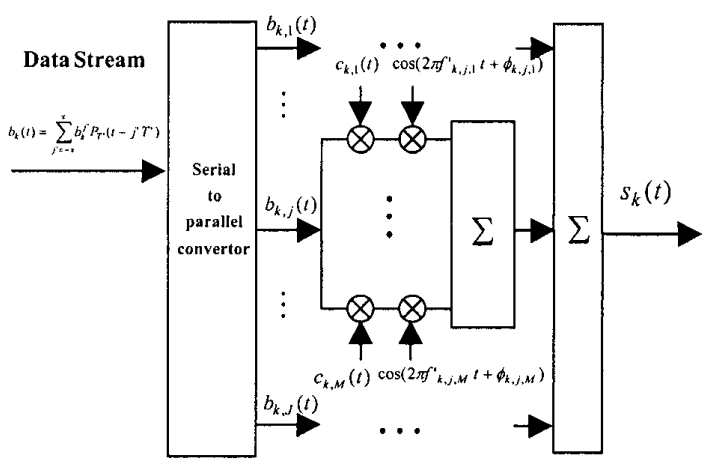

Figure 4 OFCDMA scheme 